\title{
Generation and Characterization of the Anp32e-Deficient Mouse
}

\author{
Patrick T. Reilly ${ }^{1,2}$, Samia Afzal ${ }^{2,3}$, Andrew Wakeham², Jillian Haight ${ }^{2}$, Annick You-Ten ${ }^{2}$, Kathrin \\ Zaugg $^{2 \times a}$, Joanna Dembowy ${ }^{2 \times b}$, Ashley Young ${ }^{2}$, Tak W. Mak ${ }^{1,2 *}$
}

1 Department of Cellular and Molecular Research, National Cancer Centre Singapore, Singapore, Singapore, 2 Campbell Family Cancer Research Institute, University Health Network, Toronto, Ontario, Canada, 3 Graduate Program in Immunology, University of Toronto, Toronto, Ontario, Canada

\begin{abstract}
Background: Accumulated literature suggests that the acidic nuclear phosphoprotein 32 kilodalton (Anp32) proteins control multiple cellular activities through different molecular mechanisms. Like other Anp32 family members, Anp32e (a.k.a. Cpd1, PhaplII) has been conserved throughout vertebrate evolution, suggesting that it has an important function in organismal survival.

Principal Findings: Here, we demonstrate that the Anp32e gene can be deleted in mice without any apparent effect on their wellbeing. No defects in thymocyte apoptosis in response to various stresses, fibroblast growth, gross behaviour, physical ability, or pathogenesis were defined. Furthermore, combined deletion of Anp32a and Anp32e also resulted in a viable and apparently healthy mouse.
\end{abstract}

Significance: These results provide evidence that significant functional redundancy exists among Anp32 family members.

Citation: Reilly PT, Afzal S, Wakeham A, Haight J, You-Ten A, et al. (2010) Generation and Characterization of the Anp32e-Deficient Mouse. PLoS ONE 5(10): e13597. doi:10.1371/journal.pone.0013597

Editor: Alfred Lewin, University of Florida, United States of America

Received April 20, 2010; Accepted October 1, 2010; Published October 26, 2010

Copyright: (C) 2010 Reilly et al. This is an open-access article distributed under the terms of the Creative Commons Attribution License, which permits unrestricted use, distribution, and reproduction in any medium, provided the original author and source are credited.

Funding: This research was partially supported by the Prostate Cancer Research Foundation of Canada and the U.S. Department of Defense award number PC020806. The funders had no role in study design, data collection and analysis, decision to publish, or preparation of the manuscript.

Competing Interests: The authors have declared that no competing interests exist.

*E-mail: tmak@uhnres.utoronto.ca

aa Current address: Department of Radiation Oncology, University Hospital Zürich, Zürich, Switzerland

ab Current address: Samuel Lunenfeld Research Institute, Toronto, Ontario, Canada

\section{Introduction}

The acidic nuclear phosphoprotein $\underline{32}$ kilodalton (Anp32) proteins are metazoan-specific factors that have been implicated in the regulation of a panoply of cellular functions, including proliferation $[1,2,3,4,5]$, apoptosis $[6,7,8,9,10,11,12,13]$, and differentiation $[14,15,16,17,18]$. Diverse Anp32 family members exert their functions by modulating phosphatase activity [19,20,21], mRNA stability [22,23,24], intracellular transport $[22,25,26]$, caspase activation $[8,9,10,11]$, and/or chromatin modification $[27,28,29]$. Based on this accumulated literature, it is probable that the Anp32 factors control multiple cellular activities in a broad range of physiological contexts.

The mammalian Anp32 family is comprised of Anp32a (also known as LANP, pp32, I2PP2A, or PHAPIa), Anp32b (a.k.a. APRIL, PAL31, or PHAPIb), and Anp32e (a.k.a. Cpdl or PhapIII) [30,31]. All Anp32 proteins share two regions of amino acid conservation: the N-terminal leucine-rich repeat (LRR) domain and the C-terminal acidic tail. Although the Anp32 LRR domain resembles the protein-protein interaction domains of many other protein families, the Anp32 acidic tail is highly unusual in its amino acid composition [31]. The Anp32 acidic tail contains $>100$ amino acids, of which $>80 \%$ are glutamate or aspartate residues. However, this sequence is not unique to the Anp32 family, as short conserved acidic regions resembling the
Anp32 acidic tail can be found in the Anp32-binding partner the SET translocation protein [31].

Anp32e is the least well-characterized member of the mammalian Anp32 family. Anp32e was originally cloned as a gene upregulated in the mouse brain upon postnatal development, which led to suggestions that it might play a role in neuronal proliferation and differentiation [16]. Anp32e was also identified as having a potential oncogenic function in gastric cancer [32] and myeloma [33], but then was linked to favorable treatment outcomes in patients with follicular lymphoma [34]. Biochemical studies have indicated that Anp32e is a positive regulator of caspase activation in the apoptosome. Like other Anp32 family members, Anp32e can reduce the dATP required during apoptosome formation to conform with physiological levels of this nucleotide [9].

Despite the evolutionary conservation of Anp32 family members among metazoans, mutation of the Anp32 genes does not appear to affect embryogenesis. Flies bearing a validated mutation of mapmodulin, the Drosophila homologue of Anp32, are viable [35]. In mice, deletion of Anp32a results in no detectable health deficits or defects in neuronal function [36]. In humans, no mutations in any Anp32 family members have been linked to hereditary disorders. This lack of phenotype across multiple species has led to the hypothesis that the three mammalian Anp32 orthologues are functionally redundant such that no one factor is essential for development. 
In this study, we confirm that the Anp32e gene can be deleted in mice without any apparent effect on their wellbeing. No defects in cellular growth, apoptosis, or neurological functions could be found. Furthermore, combined deletion of Anp32a and Anp32e also resulted in a viable and apparently healthy mouse. These results provide strong evidence that significant functional redundancy exists among Anp32 family members.

\section{Results}

\section{Generation of Anp32e-deficient mice}

To determine whether Anp32e had a unique biological role, we generated a targeting construct that resulted in the constitutive elimination of Anp32e exons 2-5 in one clone of murine ES cells (Figure 1A). Southern blotting of fetal mouse DNA using flanking probes confirmed the deletion of exons 2-5 (Figure 1B). Mutant mice were generated by standard blastocyst injection and backcrossed for six generations into the C57BL6 background. Quantitative RT-PCR analysis of mRNA from MEFs and thymocytes from Anp32 $\mathrm{e}^{+/+}$and Anp32 $\mathrm{e}^{-/-}$mice showed that no transcripts were expressed from the targeted Anp32e allele (Figure 1C). Using the same cDNA preparations to test for Anp32a and Anp32b mRNA expression, we found that these transcripts may be induced by deletion of Anp32e, particularly in thymocytes (Figure 1G). To more closely examine this potential induction, we tested whether protein levels of Anp32a and Anp32b were also increased in Anp32 $\mathrm{e}^{-1-}$ thymocytes. As shown in Figure 1D, there was no evident compensatory induction of either Anp32a or Anp32b protein levels in the absence of Anp32e.

We attempted to generate monoclonal and polyclonal antisera specific for Anp32e to confirm the loss of Anp32e protein in our mutant mice. Unfortunately, none of the antisera tested produced a positive signal of the expected size when used in standard immunoblotting. Procurement of additional antisera and oligobodies courtesy of Dr. Martín Radrizzani [16] resulted in western blot bands of identical sizes in extracts of Anp32 $\mathrm{e}^{+/+}$and Anp32 $\mathrm{e}^{-1-}$ MEFs and splenocytes (data not shown), suggesting that the protein detected here and in previous reports was not Anp32e.

\section{Anp32e-deficient mice are viable and fertile}

A colony of Anp32 $\mathrm{e}^{+/-}$mice of mixed genetic background was established to determine the phenotypic impact of loss of Anp32e function. Interbreeding of Anp32 $\mathrm{e}^{+/-}$mice produced Anp32 $\mathrm{e}^{-/-}$ mice at the expected Mendelian ratio, at least at time of weaning (3-4 weeks of age; Table 1). When mice in this colony were examined for fertility, we found that both male and female Anp32 $\mathrm{e}^{-/-}$mice of $6-40$ weeks of age were able to produce normal-sized litters at normal frequencies when bred to their Anp32 $2^{+/-}$counterparts (data not shown). These findings suggest that loss of Anp32e function does not deleteriously affect the development or fertility of mice under normal laboratory conditions.

\section{Anp $32 \mathrm{e}^{-/-}$cells show normal rates of proliferation and apoptosis}

Because the Anp32 proteins have been linked to cell proliferation, we examined whether there was any difference in proliferation rates between Anp3 $\mathrm{e}^{+/+}$and Anp32 $\mathrm{e}^{-/-}$cells. We isolated primary MEFs from Anp32 $\mathrm{e}^{+/+}, \operatorname{Anp} 32 \mathrm{e}^{+/-}$and Anp32 $\mathrm{e}^{-1-}$ mice from a single litter of C57/BL6 congenic animals and examined their growth in vitro. No differences were detected in a two-day analysis (Figure 2A). Furthermore, an absence of Anp32e did not alter the kinetics of release of MEFs from serum starvation-induced arrest (data not shown). These data suggest that Anp32e does not play a unique role in proliferation under either normal or low serum conditions.

Anp32e has been identified as an activator of apoptosomemediated proteolysis [9]. To test whether Anp32e-deficient cells were defective in intrinsic apoptotic signaling, we isolated thymocytes from Anp32 $\mathrm{e}^{+/+}$and Anp32 $\mathrm{e}^{-/-}$mice and exposed them to various apoptotic stimuli. As shown in Figure 2B, these thymocytes responded in equivalent fashion to both intrinsic and extrinsic apoptotic stimuli, suggesting that Anp32e does not play a unique role in apoptosis.

\section{Anp32e-deficient mice demonstrate no apparent neurological defects}

It has been suggested that Anp32 proteins might play roles in brain development [16,37]. To test this hypothesis in our mutant mice, we first performed gross histological examinations of the brains of C57BL/6-congenic Anp32 $\mathrm{e}^{-/-}$and Anp32 $\mathrm{e}^{+/+}$mice. We found no structural differences between wild-type and mutant brains, and the sizes and cellularity of various regions of these brains were comparable (data not shown). We then tested Anp32 $\mathrm{e}^{-/-}$and Anp32 $\mathrm{e}^{+/+}$mice for stimulatory responses in qualitative examinations and found that hearing and whisker stimulation evoked similar responses in both strains. Finally, we examined the relative abilities of these animals to perform in a timed Rotarod suspension assay. There was no statistically significant difference between the mean time that Anp32 $\mathrm{e}^{-1-}$ mice were able to remain suspended (126 sec) and the mean suspension time of Anp32 $\mathrm{e}^{+/+}$littermates (151 sec; Figure 3). Thus, a lack of Anp32e does not impair balance and motor control in mice.

\section{Anp32e-deficient mice demonstrate no specific pathologies}

Because our Anp32e-deficient mice showed no apparent deficits in any of the functions proposed for Anp32e, we expanded our phenotypic analyses and used standard assays of blood chemistry and hematology to perform a broad-spectrum analysis of internal organ function. No statistically significant differences between sexmatched Anp32 $\mathrm{e}^{+/+}$and Anp32 $\mathrm{e}^{-/-}$littermates at 4-8 weeks of age were detected in clinical chemistry (Table 2, top), suggesting that the functions of the liver [as defined by albumin, alanine transaminase (ALT), and aspartate transaminase (AST) levels], the heart [as defined by creatine kinase $(\mathrm{CK})$ and lactate dehydrogenase $(\mathrm{LDH})$ levels], the pancreas [as defined by amylase and glucose levels], and the kidney [as defined by creatinine, uric acid, and ion levels] are not significantly affected by loss of Anp32e. Furthermore, gross hematological examination demonstrated no differences in leukocyte or erythrocyte counts (Table 2, bottom), suggesting that Anp32e deficiency has no effect on basic blood constituents. When heart function was examined using ECG, the results obtained for Anp32 $\mathrm{e}^{-/-}$mice were within normal variability (data not shown).

To search for a function of Anp32e in preventing disease, we allowed Anp32 $\mathrm{e}^{+/-}$and Anp32 $\mathrm{e}^{-/-}$littermate mice of a mixed background to age and monitored them for susceptibility to early disease onset. We found that 5/6 Anp32 $\mathrm{e}^{-/-}$mice were still alive at 80 weeks of age when the experiment was terminated; this proportion was similar to that in the Anp32 $\mathrm{e}^{+/-}$group (data not shown). None of the mutants showed any gross defects or tissue pathologies. These results indicate that either the function of Anp32e is redundant with that of another molecule, or that its unique function will only be revealed by the application of an unusual stress yet to be determined. 


\section{A}

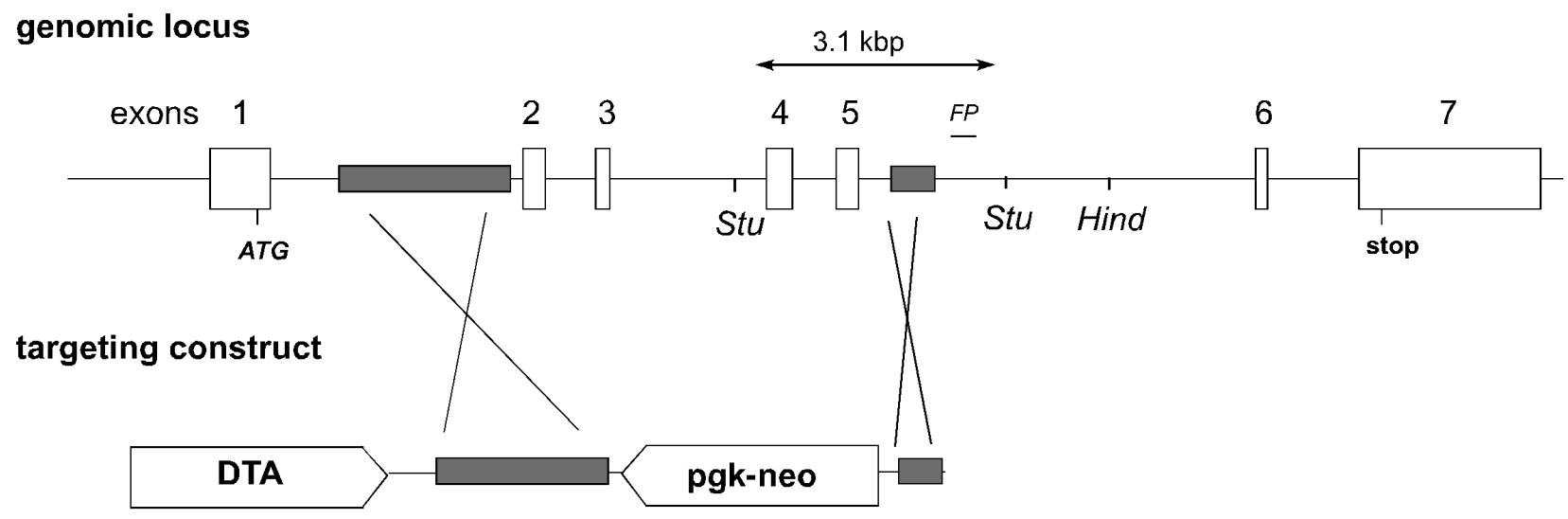

\section{targeted allele}

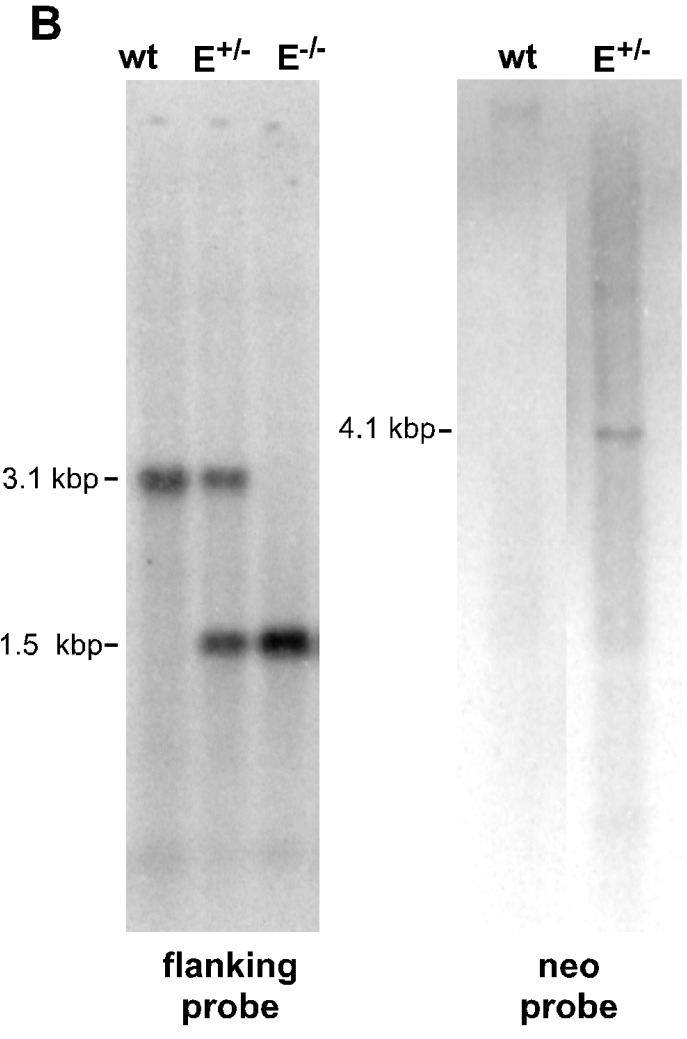

C Thymocytes

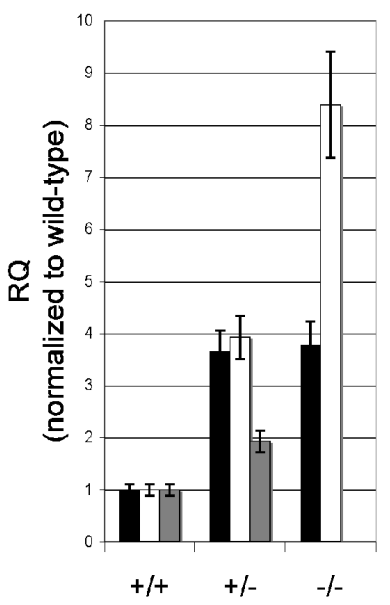

D

\section{Embryo Fibroblasts}

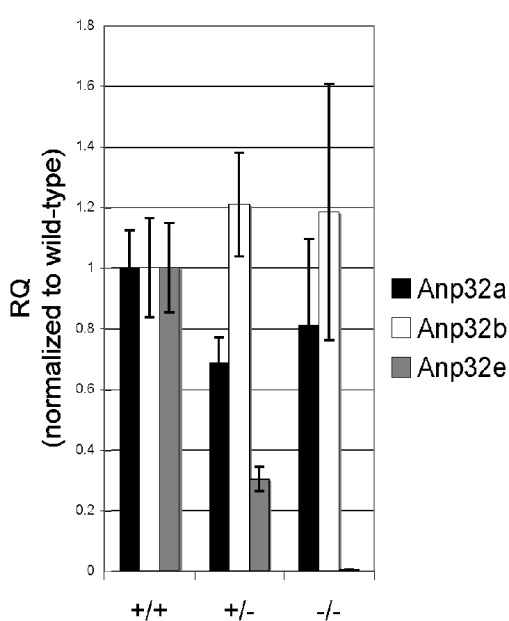

Genotype

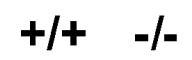

anti-Anp32a

anti-Anp32b

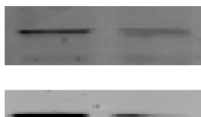

anti-tubulin

Figure 1. Generation and Validation of Anp32e-deficient mice. A. Targeting of the Anp32e gene. The diagram shows the murine genomic Anp32e locus and the targeting construct that replaced exons 2 to 6 with a pgk-neo cassette. Regions of homology in the targeting construct are shaded. Stu, Stul sites used for Southern blotting. Hind, Hindlll sites used for Southern blotting of neo. FP, flanking probe. The sizes of the diagnostic Stu1 fragments for the wild-type Anp32e allele $(3.1 \mathrm{~kb})$ and the targeted Anp32e allele $(1.5 \mathrm{~kb})$ as well as the diagnostic Hindlll fragment for neo

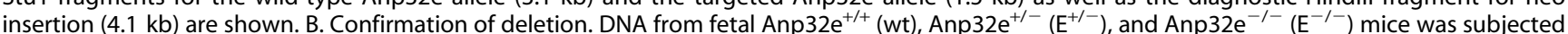

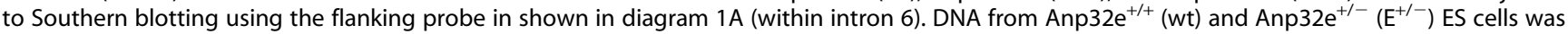
also subjected to southern blotting with a neo probe in order to confirm a single vector insertion. C. Validation of Anp32e mRNA deficiency.

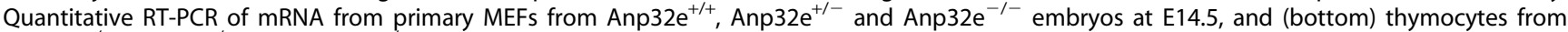
Anp32 $^{+/+}$, Anp32 $\mathrm{e}^{+/-}$and Anp32 $\mathrm{e}^{-/-}$mice at 4-8 weeks of age. Expression levels of Anp32a (black bars), Anp32b (grey bars), and Anp32e (white 
bars) mRNAs are shown relative to levels in Anp32 $\mathrm{e}^{+/+}$mice. Error bars represent the standard deviation from the mean across three technical replicates per sample. D. Absence of compensatory induction of other Anp32 proteins. Protein extracts from lymphocytes of Anp32e+/+ (wt) and Anp32e-/- (E-/ $)$ mice were probed for Anp32a, Anp32b, and beta-tubulin expression. doi:10.1371/journal.pone.0013597.g001

\section{Double Anp32a;Anp32e deficiency does not affect viability}

In a further attempt to delineate Anp32e's physiological role, we decided to create double mutant Anp32 $\mathrm{e}^{-1-} ;$ Anp32a $\mathrm{a}^{-1-}$ mice. First, we generated a novel strain of Anp32a $\mathrm{a}^{-1-}$ mice (Figure 4). Deletion of the genomic sequence was confirmed by Southern blotting using a flanking probe as well as a neomycin probe (Figure 4B). Anp32a protein deletion was confirmed by immunoblot analysis of liver and spleen extracts using a polyclonal antiAPRIL antibody [23] that crossreacts with Anp32a (Figure 4C). Consistent with a previous report [36], we found no difference in viability of the Anp32a mutation in either a mixed or backcrossed C57BL6 background (data not shown). To examine potential physiological changes in Anp32a-deficient cells, we performed growth curve analysis on early-passage mixed-bred Anp32a-/embryo fibroblasts (Figure 5). We found no significant difference in net proliferation in this analysis.

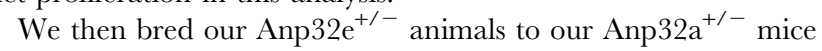
to generate double mutant progeny of a mixed 129Ola:C57BL6 background, and intercrossed the double heterozygotes to examine all potential genotypes. We found that the Anp32 $\mathrm{e}^{-/-}$genotype was present at the expected Mendelian ratio in both in the Anp32a $\mathrm{a}^{+/-}$and Anp32a $\mathrm{a}^{-/-}$backgrounds (Table 3). In fact, no statistically significant deviations from expected Mendelian ratios were observed for this breeding in its entirety. Investigation of a small number of litters showed that both males and females of the Anp32a $\mathrm{a}^{-1-} ;$ Anp32 $\mathrm{e}^{-/-}$genotype were fertile and able to produce litters of normal size and gross phenotype.

\section{Discussion}

Like other Anp32 family members, Anp32e has been conserved throughout vertebrate evolution, suggesting that it has an important function in organismal survival. However, our study demonstrates that ablation of Anp32e in mice does not affect their development or general health. Even the application of apoptotic stresses, behavioural tests, and aging experiments failed to elicit a phenotype for Anp32e-deficient animals. Although we do not claim that our analyses were exhaustive, we have performed a broad range of experiments designed to reveal effects on all currently reported functions of Anp32e.

Our work has raised an issue with respect to interpreting prior studies on Anp32e, particularly those based on the use of antibodies and oligobodies. Our investigation showed that tissues

Table 1. Expected and attained Mendelian ratios of progeny derived from the intercrossing of Anp32 $\mathrm{e}^{+/-}$mice.

\begin{tabular}{|c|c|c|c|}
\hline & \multicolumn{3}{|l|}{ genotype } \\
\hline & Anp32e $e^{+/+}$ & Anp32e $\mathrm{e}^{+\prime-}$ & Anp32e $\mathrm{e}^{-1-}$ \\
\hline expected & $8.5(25 \%)$ & 17 (50\%) & $8.5(25 \%)$ \\
\hline attained & $10(29.4 \%)$ & $16(47.0 \%)$ & $8(23.5 \%)$ \\
\hline
\end{tabular}

Data shown are the number of mice of a given genotype (percentage of total progeny). Chi square analysis determined that there was no significant difference from expected ratios.

doi:10.1371/journal.pone.0013597.t001 from a mutant mouse whose Anp32e deficiency was validated by quantitative RT-PCR produced an identical band pattern to that of wild-type tissues upon western blotting. Such a result suggests that the antibody and oligobody used in previous publications $[16,19]$ may not recognize Anp32e at all but rather an unknown factor(s).

Anp32e is the second Anp32 family member whose deletion in mice results in no apparent phenotype. Two independent loss-offunction alleles of Anp32a have now been tested in mice and have yielded no phenotype [36]. These findings all point to a probable functional redundancy of Anp32 genes, consistent with the conservation of specific protein domains among family members.

Although functional redundancy with other Anp32 family members is one possible explanation for the survival of Anp32 single mutants, the conservation of all three factors across species suggests that these genes do indeed have individual independent functions. The in vivo function(s), as elucidated from knockout analyses, may only be activated in response to stresses that remain

A

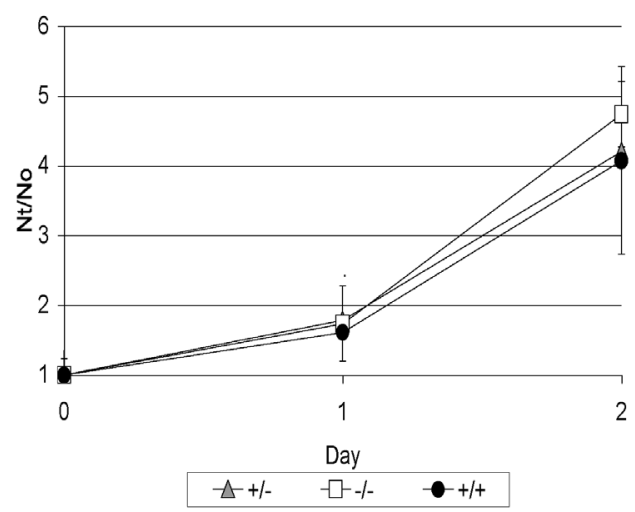

B

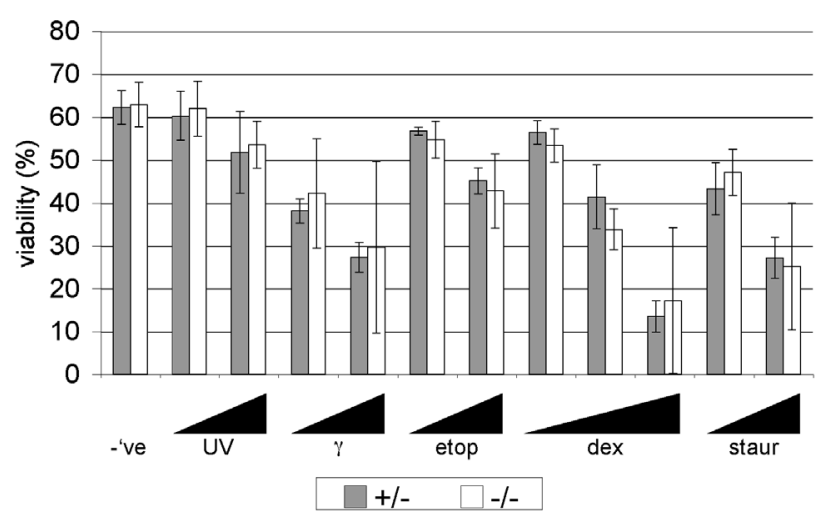

Figure 2. Normal proliferation and apoptosis of Anp32e $\mathrm{e}^{-1-}$

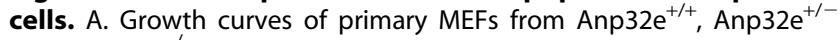
and Anp32 $\mathrm{e}^{-/-}$mice $(\mathrm{n}=2 /$ genotype). $\mathrm{Nt} / \mathrm{NO}$, cell number at time point/cell number on day 0. B. Apoptosis of Anp32e $\mathrm{e}^{+/-}$and Anp32 $\mathrm{e}^{-/-}$ thymocytes after 20 hours in culture response to (left to right): -ve, untreated controls, UV-irradiation $\left(30 \mathrm{~mJ} / \mathrm{cm}^{2}\right.$ and $\left.60 \mathrm{~mJ} / \mathrm{cm}^{2}\right), \gamma$ irradiation ( $1 \mathrm{~Gy}$ and $2 \mathrm{~Gy}$ ), etoposide $(1 \mu \mathrm{M}$ and $3 \mu \mathrm{M})$, dexamethasone ( $3 \mathrm{nM}$ and $10 \mathrm{nM})$, and staurosporine $(1 \mu \mathrm{M}$ and $3 \mu \mathrm{M})$. Results shown are mean $\%$ viable cells \pm SD ( $n=3$ /genotype).

doi:10.1371/journal.pone.0013597.g002 


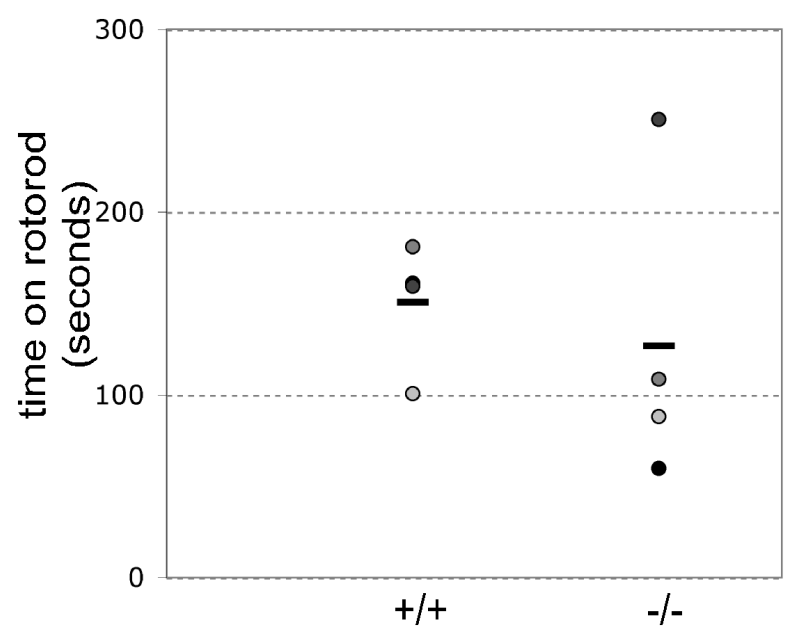

Figure 3. Normal rotorod behaviour. Individual Anp32 $\mathrm{e}^{+/+}$and Anp $32 \mathrm{e}^{-1-}$ mice (spherical symbols) were placed on a rotating rotorod and the time they remained suspended was measured in seconds. Horizontal bars, mean duration per genotype.

doi:10.1371/journal.pone.0013597.g003

to be identified. Alternatively, it may be that a complete functional overlap of the Anp32 genes exists to ensure that potentially catastrophic developmental errors are overcome.

\section{Materials and Methods}

Mice

Mice were maintained under SPF conditions in individually ventilated cages and fed 5\% irradiated meal. Unless otherwise noted, all Anp32 $32^{-/-}$mice analyzed in this study were derived from a single clone of embryonic stem (ES) cells and backcrossed six generations into the congenic C57/BL6 background.

\section{Plasmids and primers}

Sequences directing the expression of Diphtheria toxin A in mouse ES cells were cloned into pBluescript (pgk-neo) [38] to give the plasmid pBSneoDTA. Regions of the Anp32e gene adjacent to the targeted exons were cloned by high fidelity PCR. The primers GGGGTCGACCGTGAAATCGAGGGACAGGGGTCCGC and GGGGGATCCCAGACCCTTCGGTGTCTAAGCATTCTAAACGCATG were used to clone the upstream arm of homology between the SalI and BamHI sites. The primers GGGCGGCGTAAACTGGCGTTGAGGTGAGACGAGTGG and GGGGCGGCGGGGAGTGCTGGGATTAAAGGGGTGGGCGACG were used to clone the downstream arm of homology between the XbaI and NotI sites. Homologous regions of Anp32a adjacent to the targeted exons were cloned as the EcoRV-XbaI fragment upstream of exon 2 and the XbaI-EcoRV fragment downstream of exon 4.

\section{Gene targeting}

Targeting constructs were linearized and transfected by electroporation into E14K mouse ES cells as previously described [39]. Homologous recombinants were selected by culture in $0.3 \mathrm{mg} / \mathrm{ml}$ G418 for 10-14 days and screened for appropriate integration by PCR and southern blotting as previously described [38,39].

\section{Southern blotting}

Southern probes used to detect Anp32 genomic sequences were amplified from genomic ES DNA. Primer pair sequences were as follows: Anp32e, CGTGTGAATGTGTGGCGTGTGAATGTAG
Table 2. Clinical chemistry and hematology comparisons of 4

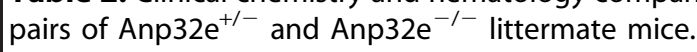

\begin{tabular}{|c|c|c|c|}
\hline & & Anp32e $e^{+/+}$ & Anp32e $\mathrm{e}^{-1-}$ \\
\hline \multirow{14}{*}{$\begin{array}{l}\text { Clinical } \\
\text { chemistry }\end{array}$} & Albumin (g/L) & $35.3 \pm 1.7$ & $35.5 \pm 1.7$ \\
\hline & ALT (Units/L) & $25.8 \pm 6.0$ & $31.6 \pm 3.6$ \\
\hline & AST (Units/L) & $50.8 \pm 4.3$ & $53.0 \pm 5.6$ \\
\hline & CK (Units/L) & $192.5 \pm 92.4$ & $373.8 \pm 211.7$ \\
\hline & LDH (Units/L) & $397.3 \pm 48.9$ & $466.0 \pm 80.3$ \\
\hline & Amylase (Units/L) & $2478.8 \pm 358.3$ & $2604.5 \pm 515.6$ \\
\hline & Cholesterol (mM) & $3.0 \pm 0.6$ & $2.9 \pm 0.7$ \\
\hline & Triglycerides (mM) & $0.7 \pm 0.1$ & $0.7 \pm 0.2$ \\
\hline & Glucose (mM) & $12.9 \pm 1.1$ & $11.1 \pm 1.3$ \\
\hline & Creatinine (c) & $16.3 \pm 1.5$ & $11.3 \pm 5.1$ \\
\hline & Uric Acid $(\mu \mathrm{M})$ & $65.8 \pm 18.6$ & $61.5 \pm 19.2$ \\
\hline & $\mathrm{Na}^{+}(\mathrm{mM})$ & $147.0 \pm 1.8$ & $145.0 \pm 2.0$ \\
\hline & $\mathrm{K}^{+}(\mathrm{mM})$ & $5.4 \pm 0.3$ & $5.5 \pm 0.6$ \\
\hline & $\mathrm{Cl}^{-}(\mathrm{mM})$ & $107.5 \pm 1.7$ & $107.0 \pm 0.0$ \\
\hline \multirow[t]{8}{*}{ Hematology } & RBC (billions/mL) & $11.1 \pm 0.5$ & $11.1 \pm 0.3$ \\
\hline & $\mathrm{HgB}(\mathrm{g} / \mathrm{L})$ & $166.8 \pm 7.8$ & $161.5 \pm 6.2$ \\
\hline & $\mathrm{HCT}(\mathrm{L} / \mathrm{L})$ & $0.49 \pm 0.02$ & $0.48 \pm 0.02$ \\
\hline & MCV (fL) & $43.7 \pm 0.3$ & $43.0 \pm 0.9$ \\
\hline & MCH (pg/cell) & $15.0 \pm 0.2$ & $14.6 \pm 0.3$ \\
\hline & MCHC (g/L) & $342.3 \pm 3.0$ & $340.0 \pm 3.4$ \\
\hline & PLT (millions/mL) & $1146 \pm 200$ & $1221 \pm 70$ \\
\hline & WBC (millions/mL) & $15.6 \pm 0.9$ & $15.3 \pm 0.7$ \\
\hline
\end{tabular}

Data shown are the mean levels of the indicated parameters $\pm S D(n=4 /$ genotype). $A L T$, Alanine transaminase. AST, Aspartate transaminase. $C K$, Creatine kinase. $L D H$, Lactose dehydrogenase. $\mathrm{Na}^{+}$, Sodium. $\mathrm{K}^{+}$, Potassium. $\mathrm{Cl}^{-}$, Chloride. $H g B$, Hemoglobin. $H C T$, Hematocrit. $M C V$, Mean corpuscular volume. $M C H$, Mean corpuscular hemoglobin. $M C H C$, Mean corpuscular hemoglobin concentration. PLT, Platelet count. WBC, White blood cell count.

doi:10.1371/journal.pone.0013597.t002

and TTTCGCACTCAATGCACTGGCTCTTAGA; Anp32a, CGATGGATTCTGTGACCCTTCAGCG and CACAAGTCACGTACCATTGCACCC. Genomic DNA was digested, resolved on gels, transferred to nylon membranes, and hybridized to the above probes using standard procedures [38,39]. To optimize image sizing, some figures have been adjusted so that desired lanes appear beside one another.

\section{Quantitative RT-PCR}

RNA from adult mouse tissues was isolated using a commercial kit and protocol (Qiagen). cDNA was reverse-transcribed from $1 \mu \mathrm{g}$ mRNA using Superscript II enzyme (Invitrogen), and analyzed by Syber-Green nucleotide incorporation in separate PCR reactions. Primers were:

Gapdh (loading control), AACAGGAAGCCGATCACATCTT and GCCCTTCGACAATGCGAAAGTT; Anp32a, GCGAAAACAGAATCTCAGGGG and TTCTTCAGGGGGTCTATTGTG; Anp32b, AGCGGTTCGAGAACTTGTCTT and CAGGTTATTGGCACTTAGGTTCA; Anp32e, AATTGCTTGTGTGTCAATGGGG and GGCGATGCTAAGAAACTCCAG.

\section{Western blotting}

Immunoblotting to detect Anp32a was performed on $5 \mu \mathrm{g}$ protein extracted by NP-40 detergent lysis of homogenized mouse 


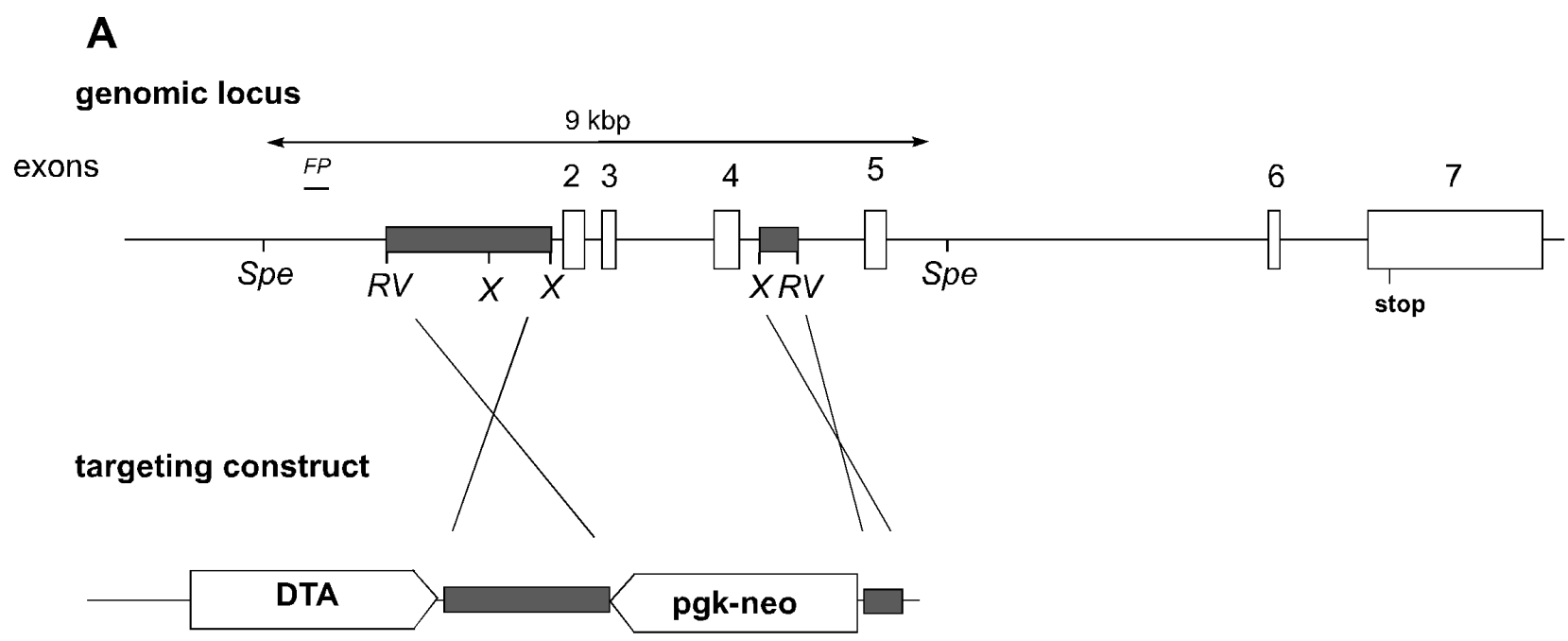

targeted allele

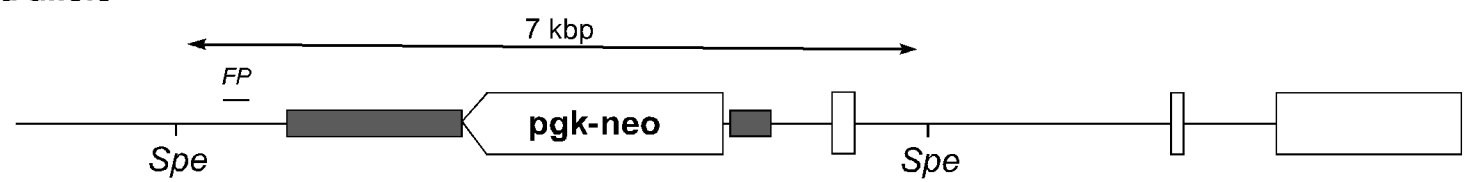

B

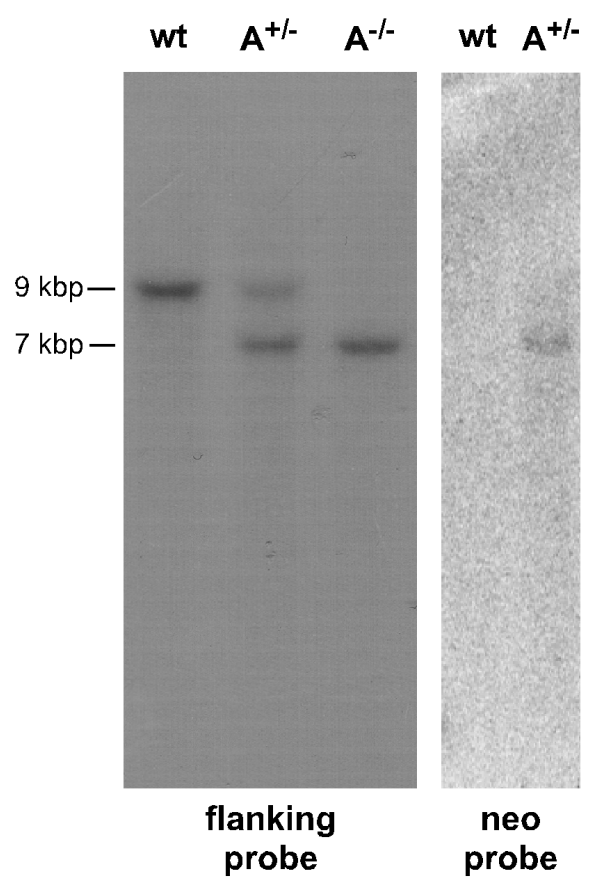

C

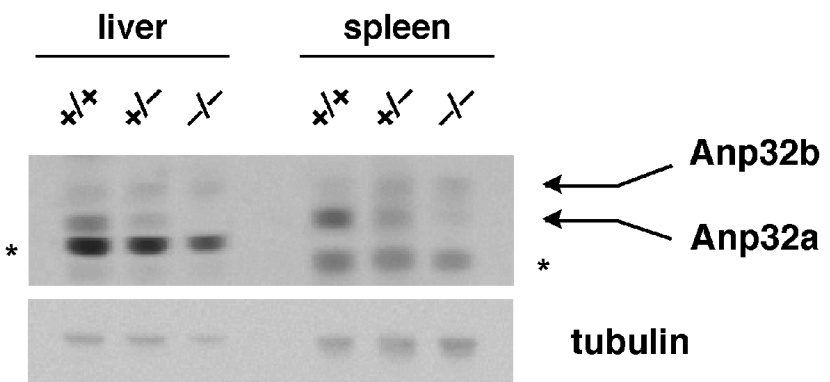

Figure 4. Generation and Validation of Anp32a-deficient mice. A. Targeting of the Anp32a gene. The diagram shows the murine genomic Anp32a locus and the targeting construct that replaced exons 2 to 5 with a pgk-neo cassette. Regions of homology in the targeting construct are shaded. RV, EcoRV; X, Xbal; Spe, Spel; $F P$, flanking probe used for Southern blot analysis. B. Confirmation of deletion. DNA from embryos of Anp32a ${ }^{+/+}$ (wt), Anp32a $\mathrm{a}^{+-}\left(\mathrm{A}^{+/-}\right)$and Anp32a $\mathrm{a}^{-/-}\left(\mathrm{A}^{-/-}\right)$was subjected to Southern blotting using the flanking probe in $\mathrm{A}$ (within intron 1). Anp32a $\mathrm{a}^{+/+}(\mathrm{wt})$ and Anp32a $a^{+/-}\left(\mathrm{A}^{+/-}\right)$ES-cell DNA was also probed for the neomycin resistance gene (Neo probe). C. Validation of Anp32a protein deficiency by immunoblotting of extracts from mouse liver and spleen. Primary anti-APRIL antibody raised in mice against recombinant human Anp32b protein recognizes both the murine Anp32b protein and the Anp32a protein, which can be distinguished by size. Tubulin, loading control. ${ }^{*}$, non-specific bands seen in liver and spleen.

doi:10.1371/journal.pone.0013597.g004 


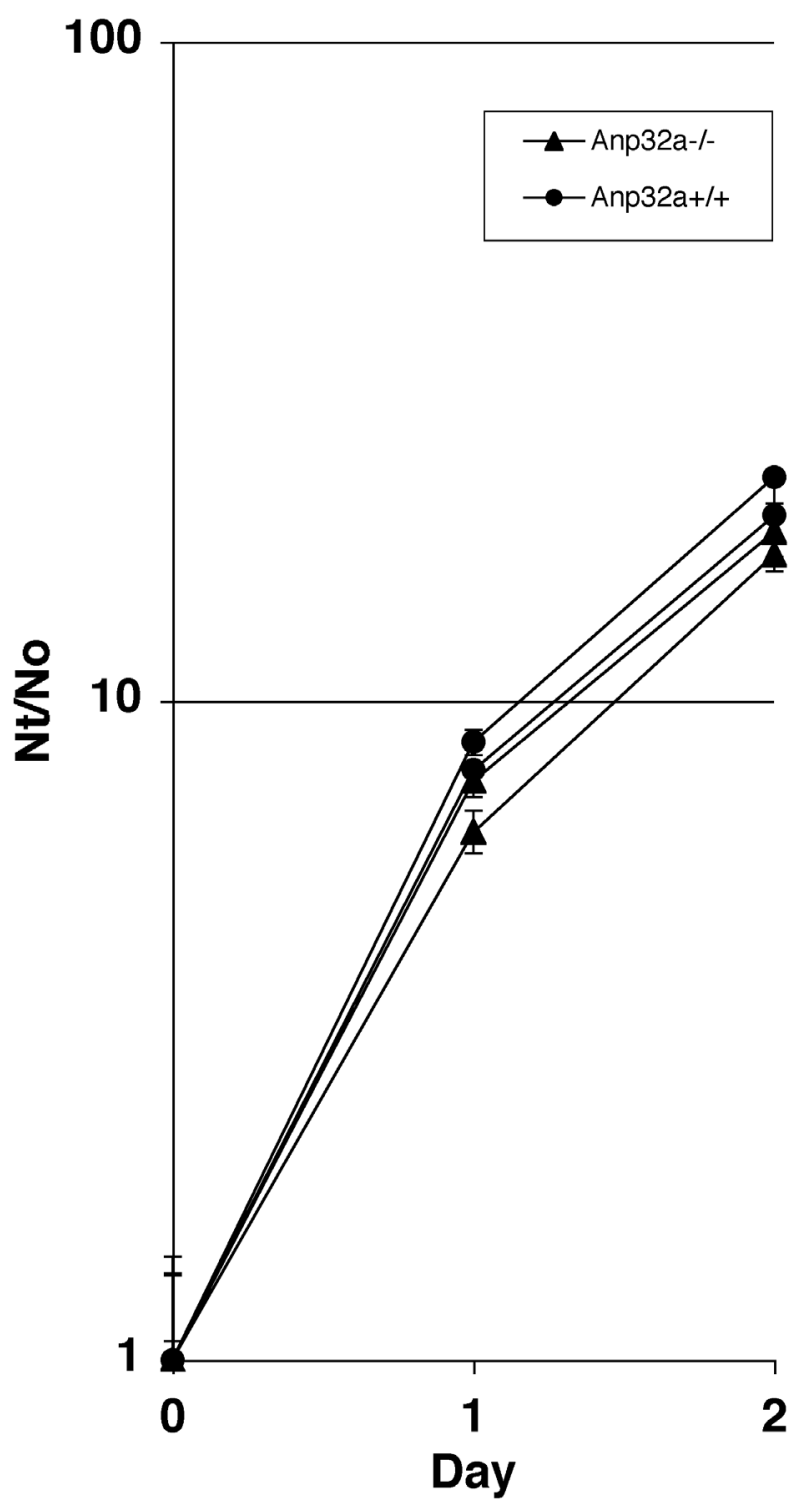

Figure 5. Growth Curve of Anp32a-deficient fibroblasts. Growth curves of early passage primary MEFs from littermate isolates of Anp32a $\mathrm{a}^{+/+}$and Anp32a $\mathrm{a}^{-/-}$embryos. Two isolates per genotype to give four littermate MEF lines total. Nt/NO, cell number at time point/cell number on day 0 .

doi:10.1371/journal.pone.0013597.g005 thymuses, livers, or spleens. After SDS-PAGE resolution, proteins were transferred to PDVF membranes and probed with anti- $\beta$ tubulin (loading control; Li-Cor Bioscience), anti-APRIL antibody that detected both the Anp32a and Anp32b proteins (courtesy of Dr. Imed Gallouzi, McGill University, Montreal, Canada) [23], anti-Anp32a (sc-5652, Santa Cruz Biotech), or anti-Anp32b (10843-1-AP, Proteintech). Bands on blots were visualized according to Li-Cor Odyssey standard protocols.

\section{Rotorod analysis}

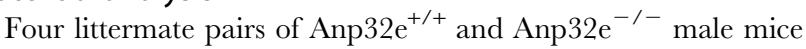
(4-8 weeks old) were examined for their ability to stay on the Economex Rota Rod when the apparatus was operating at an initial speed of $4 \mathrm{rpm}$ that subsequently increased at a rate of $0.1 \mathrm{rpm} / \mathrm{second}$. Each mouse was tested three times with a $15 \mathrm{~min}$ rest period between tests.

Clinical chemistry, hematology and electrocardiography

Blood was extracted from the saphenous vein of 4 pairs of

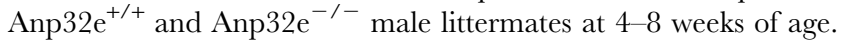
Samples were sent to IDEXX Laboratories Inc. for commercial analysis of wide spectrum clinical chemistry. Additional blood samples from the same mice were tested for hematological differences using a Coulter $\mathrm{A}^{\mathrm{c}} \cdot \mathrm{T}$ diff Analyzer (Coulter Corp.). Electrocardiograms (ECG) were generated for these same mice using the Power Lab/4SP system with ML135 and MLA0112 attachments (AD Instruments). The 5 electrodes of this apparatus were connected subcutaneously at the limbs. The ECG were analyzed using the SAECG extension for Chart 4 (AD Instruments).

\section{Apoptosis}

Apoptosis assays were performed as described previously $[39,40]$. Briefly, single cell suspensions were prepared of total thymocytes from three sex-matched littermate pairs of Anp32 $\mathrm{e}^{+/+}$ and Anp32 $\mathrm{e}^{-/-}$mice (4-8 weeks old). The cells were exposed to apoptotic stimuli as detailed in Figure $2 \mathrm{~B}$ and then cultured overnight in RPMI medium containing penicillin/streptomycin, $10 \%$ fetal bovine serum (FBS) and L-glutamine. Viability was determined by propidium iodide [16] exclusion and flow cytometry using a FACScaliber (BD Pharmingen).

\section{Growth curves}

Growth curves were established as described previously [41]. Briefly, primary murine embryonic fibroblasts (MEFs) were derived from 2 embryos/genotype (Anp32 $\mathrm{e}^{+/+}$, Anp32 $\mathrm{e}^{+/-}$, Anp32 $\mathrm{e}^{-/-}$) on embryonic day 14.5 (E14.5). MEFs were cultured in Dulbecco's MEM containing penicillin/streptomycin, $10 \%$ FBS, L-glutamine, and beta-mercaptoethanol. After two passages,

Table 3. Expected and attained Mendelian ratios of progeny derived from the intercrossing of Anp $32 \mathrm{e}^{+/-} ;$Anp $32 \mathrm{a}^{+/-}$double mutant littermate mice.

\begin{tabular}{|c|c|c|c|c|c|c|c|c|c|}
\hline & \multicolumn{9}{|l|}{ Genotype } \\
\hline & $\begin{array}{l}\text { Anp32a } a^{+++} ; \\
\text {Anp32e }\end{array}$ & $\begin{array}{l}\text { Anp32a } \mathrm{a}^{+++} ; \\
\text {Anp32e }\end{array}$ & $\begin{array}{l}\text { Anp32a } a^{+++} ; \\
\text {Anp32e }\end{array}$ & $\begin{array}{l}\text { Anp32a } \mathrm{a}^{+/-} ; \\
\text {Anp32e }\end{array}$ & $\begin{array}{l}\text { Anp32a } a^{+/-} \\
\text {Anp32 }\end{array}$ & $\begin{array}{l}\text { Anp32a } \mathrm{a}^{+/-} \text {; } \\
\text { Anp32e }\end{array}$ & $\begin{array}{l}\text { Anp32a } a^{-1-} \\
\text { Anp32e }\end{array}$ & $\begin{array}{l}\text { Anp32a } \mathrm{a}^{-/-} ; \\
\text {Anp32e }^{+/-}\end{array}$ & $\begin{array}{l}\text { Anp32a } \mathrm{a}^{-I-} ; \\
\text { Anp32 }\end{array}$ \\
\hline Expected & $2(7.4 \%)$ & 3 (11.1\%) & $2(7.4 \%)$ & 3 (11.1\%) & 7 (25.9\%) & $3(11.1 \%)$ & $2(7.4 \%)$ & 3 (11.1\%) & $2(7.4 \%)$ \\
\hline Attained & 3 (11.1\%) & $6(22.2 \%)$ & 3 (11.1\%) & 3 (11.1\%) & 3 (11.1\%) & $6(22.2 \%)$ & 1 (3.7\%) & $0(0 \%)$ & $2(7.4 \%)$ \\
\hline
\end{tabular}


the cells were plated at $5 \times 10^{4}$ cells/well in 6 -well dishes. For serum deprivation studies, the medium was replaced the next day (Day 0) with medium containing $0.1 \%$ FBS. For quantitation, triplicate wells for each genotype and each time point (day 0,1 or 2) were washed in PBS and trypsinized. Cells were collected in specified volumes and counted on a hemocytometer. To eliminate small variations in seeding and plating efficiency, growth was normalized to the initial cell count on Day 0 .

\section{Ethics Statement}

All animal treatments were approved in advance by the University Health Network Animal Care Committee, protocol numbers 0985 and 02147, and performed in the Ontario Cancer Institute Animal Research Centre, Ontario registry number 0050.

\section{References}

1. Adegbola O, Pasternack GR (2005) Phosphorylated retinoblastoma protein complexes with pp32 and inhibits pp32-mediated apoptosis. J Biol Chem 280: 15497-15502.

2. Amasaki H, Ogawa M, Nagasao J, Mutoh K, Ichihara N, et al. (2003) Distributional changes of BrdU, PCNA, E2F1 and PAL31 molecules in developing murine palatal rugae. Ann Anat 185: 517-523.

3. Fan Z, Zhang H, Zhang Q(2006) Tumor suppressor pp32 represses cell growth through inhibition of transcription by blocking acetylation and phosphorylation of histone H3 and initiating its proapoptotic activity. Cell Death Differ 13: 1485-1494.

4. Malek SN, Katumuluwa AI, Pasternack GR (1990) Identification and preliminary characterization of two related proliferation-associated nuclear phosphoproteins. J Biol Chem 265: 13400-13409.

5. Sun W, Hattori N, Mutai H, Toyoshima Y, Kimura H, et al. (2001) PAL31, a nuclear protein required for progression to the $\mathrm{S}$ phase. Biochem Biophys Res Commun 280: 1048-1054

6. Beresford PJ, Zhang D, Oh DY, Fan Z, Greer EL, et al. (2001) Granzyme A activates an endoplasmic reticulum-associated caspase-independent nuclease to induce single-stranded DNA nicks. J Biol Chem 276: 43285-43293.

7. Fan Z, Beresford PJ, Oh DY, Zhang D, Lieberman J (2003) Tumor suppressor NM23-H1 is a granzyme A-activated DNase during CTL-mediated apoptosis, and the nucleosome assembly protein SET is its inhibitor. Cell 112: 659-672.

8. Hill MM, Adrain C, Duriez PJ, Creagh EM, Martin SJ (2004) Analysis of the composition, assembly kinetics and activity of native Apaf-1 apoptosomes. EMBO J 23: 2134-2145.

9. Jiang X, Kim HE, Shu H, Zhao Y, Zhang H, et al. (2003) Distinctive roles of PHAP proteins and prothymosin-alpha in a death regulatory pathway. Science 299: 223-226.

10. Schafer ZT, Parrish AB, Wright KM, Margolis SS, Marks JR, et al. (2006) Enhanced sensitivity to cytochrome c-induced apoptosis mediated by PHAPI in breast cancer cells. Cancer Res 66: 2210-2218.

11. Sun W, Kimura H, Hattori N, Tanaka S, Matsuyama S, et al. (2006) Proliferation related acidic leucine-rich protein PAL31 functions as a caspase-3 inhibitor. Biochem Biophys Res Commun 342: 817-823.

12. Hoffarth S, Zitzer A, Wiewrodt R, Hahnel PS, Beyer V, et al. (2008) pp32/ PHAPI determines the apoptosis response of non-small-cell lung cancer. Cell Death Differ 15: 161-170.

13. Pan W, da Graca LS, Shao Y, Yin Q, Wu H, et al. (2009) PHAPI/pp32 suppresses tumorigenesis by stimulating apoptosis. J Biol Chem 284: 6946-6954.

14. Anisimov SV, Tarasov KV, Tweedie D, Stern MD, Wobus AM, et al. (2002) SAGE identification of gene transcripts with profiles unique to pluripotent mouse R1 embryonic stem cells. Genomics 79: 169-176.

15. Brody JR, Kadkol SS, Hauer MC, Rajaii F, Lee J, et al. (2004) pp32 reduction induces differentiation of TSU-Pr1 cells. Am J Pathol 164: 273-283.

16. Radrizzani M, Vila-Ortiz G, Cafferata EG, Di Tella MC, Gonzalez-Guerrico A, et al. (2001) Differential expression of CPD1 during postnatal development in the mouse cerebellum. Brain Res 907: 162-174.

17. Puente LG, Carriere JF, Kelly JF, Megeney LA (2004) Comparative analysis of phosphoprotein-enriched myocyte proteomes reveals widespread alterations during differentiation. FEBS Lett 574: 138-144.

18. Kular RK, Cvetanovic M, Siferd S, Kini AR, Opal P (2009) Neuronal differentiation is regulated by leucine-rich acidic nuclear protein (LANP), a member of the inhibitor of histone acetyltransferase complex. J Biol Chem 284: 7783-7792.

19. Costanzo RV, Vila-Ortiz GJ, Perandones C, Carminatti H, Matilla A, et al. (2006) Anp32e/Cpd1 regulates protein phosphatase 2A activity at synapses during synaptogenesis. Eur J Neurosci 23: 309-324.

20. Fukukawa C, Tanuma N, Okada T, Kikuchi K, Shima H (2005) pp32/I1(PP2A) negatively regulates the Raf-1/MEK/ERK pathway. Cancer Lett 226: $155-160$.

\section{Acknowledgments}

We would like to thank Arda Shahinian, Gordon Duncan, Jennifer Silvester, Andrew Elia, Christine Chio for technical assistance, as well as Mary Saunders for scientific editing of the manuscript. The authors would also like to acknowledge the Samuel Lunenfeld Research Institute's Centre for Modeling Human Disease Mouse Phenotyping Facility for their technical screening services (www.cmhd.ca).

\section{Author Contributions}

Conceived and designed the experiments: PTR TM. Performed the experiments: PTR SA AW AYT JH KZ JD AKY. Analyzed the data: PTR AKY. Contributed reagents/materials/analysis tools: PTR AW. Wrote the paper: PTR TM.

21. Li M, Guo H, Damuni Z (1995) Purification and characterization of two potent heat-stable protein inhibitors of protein phosphatase 2A from bovine kidney. Biochemistry 34: 1988-1996.

22. Fries B, Heukeshoven J, Hauber I, Gruttner C, Stocking C, et al. (2007) Analysis of nucleocytoplasmic trafficking of the HuR ligand APRIL and its influence on CD83 expression. J Biol Chem 282: 4504-4515.

23. Gallouzi IE, Brennan CM, Steitz JA (2001) Protein ligands mediate the CRM1dependent export of HuR in response to heat shock. RNA 7: 1348-1361.

24. Higashino F, Aoyagi M, Takahashi A, Ishino M, Taoka M, et al. (2005) Adenovirus E4orf6 targets pp32/LANP to control the fate of ARE-containing mRNAs by perturbing the CRM1-dependent mechanism. J Cell Biol 170: $15-20$.

25. Itin C, Ulitzur N, Muhlbauer B, Pfeffer SR (1999) Mapmodulin, cytoplasmic dynein, and microtubules enhance the transport of mannose 6-phosphate receptors from endosomes to the trans-golgi network. Mol Biol Cell 10: 2191-2197.

26. Opal P, Garcia JJ, Propst F, Matilla A, Orr HT, et al. (2003) Mapmodulin/ leucine-rich acidic nuclear protein binds the light chain of microtubuleassociated protein $1 \mathrm{~B}$ and modulates neuritogenesis. J Biol Chem 278: 34691-34699.

27. Seo SB, Macfarlan T, McNamara P, Hong R, Mukai Y, et al. (2002) Regulation of histone acetylation and transcription by nuclear protein pp32, a subunit of the INHAT complex. J Biol Chem 277: 14005-14010.

28. Schneider R, Bannister AJ, Weise C, Kouzarides T (2004) Direct binding of INHAT to H3 tails disrupted by modifications. J Biol Chem 279: 23859-23862.

29. Cvetanovic M, Rooney RJ, Garcia JJ, Toporovskaya N, Zoghbi HY, et al. (2007) The role of LANP and ataxin 1 in E4F-mediated transcriptional repression. EMBO Rep 8: 671-677.

30. Matilla A, Radrizzani M (2005) The Anp32 family of proteins containing leucine-rich repeats. Cerebellum 4: 7-18.

31. Santa-Coloma TA (2003) Anp32e (Cpdl) and related protein phosphatase 2 inhibitors. Cerebellum 2: 310-320.

32. Tsukamoto Y, Uchida T, Karnan S, Noguchi T, Nguyen LT, et al. (2008) Genome-wide analysis of DNA copy number alterations and gene expression in gastric cancer. J Pathol 216: 471-482.

33. Walker BA, Leone PE, Chiecchio L, Dickens NJ, Jenner NW, et al. A compendium of myeloma associated chromosomal copy number abnormalities and their prognostic value. Blood.

34. Bjorck E, Ek S, Landgren O, Jerkeman M, Ehinger M, et al. (2005) High expression of cyclin $\mathrm{B} 1$ predicts a favorable outcome in patients with follicular lymphoma. Blood 105: 2908-2915.

35. Buszczak M, Paterno S, Lighthouse D, Bachman J, Planck J, et al. (2007) The carnegie protein trap library: a versatile tool for Drosophila developmental studies. Genetics 175: 1505-1531

36. Opal P, Garcia JJ, McCall AE, Xu B, Weeber EJ, et al. (2004) Generation and characterization of LANP/pp32 null mice. Mol Cell Biol 24: 3140-3149.

37. Jiang M, Ma Y, Ni X, Cao G, Ji C, et al. (2002) Molecular cloning and characterization of a novel human gene (ANP32E alias LANPL) from human fetal brain. Cytogenet Genome Res 97: 68-71.

38. Ruland J, Sirard C, Elia A, MacPherson D, Wakeham A, et al. (2001) p53 accumulation, defective cell proliferation, and early embryonic lethality in mice lacking tsg101. Proc Natl Acad Sci U S A 98: 1859-1864.

39. Okada H, Suh WK, Jin J, Woo M, Du C, et al. (2002) Generation and characterization of Smac/DIABLO-deficient mice. Mol Cell Biol 22: 3509-3517.

40. Hao Z, Duncan GS, Chang CC, Elia A, Fang M, et al. (2005) Specific ablation of the apoptotic functions of cytochrome $\mathrm{C}$ reveals a differential requirement for cytochrome C and Apaf-1 in apoptosis. Cell 121: 579-591.

41. Reilly PT, Herr W (2002) Spontaneous reversion of tsBN67 cell proliferation and cytokinesis defects in the absence of HCF-1 function. Exp Cell Res 277: $119-130$. 